\section{Mimicking mucins}

\section{By Michael J. Haas, Senior Writer}

Researchers have developed synthetic glycopolymers that model the binding behavior of mucins on the surfaces of living cells. The technique, which avoids the difficulties associated with studying mucins in their complex native state, could lead to improved cancer therapies that target mucins and other glycoproteins.

Such 'mucin mimics' could fill an important technical gap, if mimics with the functional-as well as the physical-properties of glycoproteins could be designed.

Mucins are a family of large, highly glycosylated proteins, some of which are membrane-bound cell-surface receptors with an array of signal transduction and intracellular functions. Changes in expression or degree of glycosylation of cell-surface mucins have been implicated in several cancers, including pancreatic, prostate, ovarian and

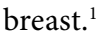

However, mucins are difficult to study in their native state. One reason is their glycan structures: the number and length of saccharide chains attached to a mucin's peptide backbone are highly variable. According to David Rabuka, coauthor of the paper in the Journal of the American Chemical Society and spokesperson for the research team, this structural heterogeneity makes it difficult to correlate mucin behavior with a specific glycan structure. Rabuka is a graduate student in the Department of Chemistry and the Department of Molecular and Cell Biology at the University of California, Berkeley.

David Gold, director of laboratory administration at Garden State Cancer Center, agreed with Rabuka. "Mucins are very complex molecules, akin to snowflakes," Gold said. "No two molecules of a single mucin species produced by a single person in a single tissue are identical."

Another challenge, Rabuka said, is that "the population or number density of a native glycoprotein on the cell surface will vary according to the life cycle of the cell," which makes it difficult to account for the total number of mucins contributing to a given level of biological activity.

In the JACS paper, the UC Berkeley researchers inserted synthetic glycopolymers into living cell membranes, where the glycopolymers mimicked the binding behavior of mucins. ${ }^{2}$ The approach allows specific chemical control over the structure and surface population of the mimics.

Rabuka said the technique overcomes both the confounding problems of structural heterogeneity and variable surface populations. The synthetic method allowed researchers to produce mucin-like glycopolymers with homogeneous structures. Moreover, he said, "we can control how much of the glycopolymer is exposed on the cell sur- face by controlling the conditions under which the glycopolymers are inserted into the cell."

The glycopolymers consisted of a poly(methyl vinyl ketone) (poly(MVK)) backbone with synthetic mono- or disaccharide glycan units attached and a phospholipid tail that allowed the glycopolymer to incorporate itself into a membrane.

"The synthesis of homogeneous glycoproteins is lengthy and difficult," Rabuka said. "The synthesis of the poly(MVK)s takes about one-tenth of the time."

The research team previously tested the poly(MVK) mucin mimics in synthetic membrane systems. ${ }^{3}$ In the current study, the team incubated the fluorophore-tagged mimics with a type of Chinese hamster ovary cell that does not naturally express mucins. The team monitored the mucin mimics with fluorescence correlation spectroscopy (FCS), an imaging technique that detects only moving fluorophores.

Imaging results showed that the mimics had comparable mobility to endogenously expressed glycosylphosphatidylinositol (GPI)-anchored proteins designed by the researchers ${ }^{4}$ - but a more consistent number density from one cell to another. Moreover, that density depended only on the incubation conditions, not on the glycan structure of the particular mimic.

Taken together, the results demonstrate that the technique provided a level of control that allowed the variables of glycan structure, density or dynamics of the mucin mimics to be modeled and explored separately.

The team was led by Carolyn Bertozzi, professor of chemistry and molecular and cell biology at UC Berkeley. Bertozzi is also director of the Molecular Foundry at the University of California's Lawrence Berkeley National Laboratory and an investigator at the Howard Hughes Medical Institute.

\section{Model behavior}

The ability to model mucin behavior in a controlled fashion could have implications for companies developing compounds that target mucins or other cell-surface glycoproteins for cancer.

An example of the latter is Arius Research Inc., which expects to start a Phase I trial of its AR001 anti-CD44 mAb to treat solid tumors in late 2008. CD44 is a cell-surface glycoprotein that plays a role in intercellular interactions as well as cell adhesion and migration. AR001 targets CD44 on cancer stem cells.

Such a technique would allow researchers in industry to begin building a much needed, systematic understanding of the relationship between a specific glycan structure and its behavior, said David Young, president and CEO of Arius.

"There are lots of carbohydrate changes in cancer cells," he said. "But it is hard to tell what effect those changes in glycosylation have, because they often occur simultaneously. This has been one of the major challenges in figuring out what role carbohydrates play in cancer."

For AR001, "the binding epitope of the $\mathrm{mAb}$ is modified by carbohydrates," Young told SciBX. "When you take away the carbohydrates from $\mathrm{CD} 44$, the $\mathrm{mAb}$ can't bind it-yet we know that the mAb doesn't bind directly to the carbohydrates.' 
Having a tool that specifically probed the behavior of carbohydrates could lead to a better understanding of what function they fulfill in cancer cells-and perhaps to the development of more effective mAbs, he said.

"Right now the technique is a good way of isolating one glycan structure from all of the others," he said. "This could be used to test inhibitors of glycan structures-for example, those which play a role in adhesion in cancer."

Juergen Hess, spokesperson for scientific affairs at Trion Pharma GmbH, said the mucin-like mobility and more consistent density of the mimics on the cell surface satisfied important prerequisites for "the study of the dynamics of protein-protein interactions in a simplified model based on unique binding partners."

Added Garden State's Gold: “The value of this technology is to create an artificial target cell that presents carbohydrate epitope structures, not only for mucins, but for other potential epitopes as well," he said. "This could be quite useful for studying the in vitro kinetics of antibody-antigen interactions."

\section{Room for improvement}

Though the technique has overcome some of the primary obstacles to the study of mucins, there are shortcomings that need to be addressed.

"At the present time, the technology is limited by the ability to synthesize carbohydrate chains of sufficient length and complexity," said Gold, noting that the researchers examined only mono- or disaccharide glycans. Natural mucins can have glycan side chains of up to 20 saccharides.

Gold did note that cancerous tissues tend to produce mucins that have fewer and shorter glycans than those in normal tissues.

"A technique that could prepare epitopes of three or four sugars-with or without branching-could provide good opportunities as a tool to study some mAbs and other carbohydratebinding molecules, such as lectins," he said.

The other drawback, said Gold, "is the lack of a natural peptide backbone," which plays a role in the intracellular signal transduction of mucins. Thus, the synthetic glycopolymers are unable to probe those functions of natural mucins.

Gold developed the PAM4 antibody against mucin 1 (MUC1; CD227) that Immunomedics Inc. has in clinical development. In January, the company began a Phase Ib trial of IMMU-107, a humanized PAM4 mAb labeled with a radioisotope of yttrium, to treat pancreatic cancer in combination with Gemzar gemcitabine from Eli Lilly and Co.

Trion's Hess noted the mimics' synthetic polymer backbone prevents them from modeling interactions that depend on a glycoprotein's underlying peptide structure-such as mAb-mucin binding. "In our field of expertise of antibody engineering, this glycopolymer technology could not be used because the relationships between antigen-antibody interaction sites and the underlying protein conformations are more complex."

According to Hess, in December partners Trion and Fresenius SE submitted an MAA for catumaxomab, a polyfunctional antibody targeting CD3 and the glycoprotein epithelial cell adhesion molecule (EpCAM), to treat malignant ascites (excess fluid in the peritoneal cavity). The partners also have the mAb in Phase II trials for gastrointesti- nal, gastric and ovarian cancers

Arius's Young agreed with Gold-and disagreed with Hess-about whether the technique could model the binding between antibodies and antigens having carbohydrate structures.

"It depends on what the antibody's binding target is," Young said. "If it's a glycolipid-some glycolipids are known cancer antigens-this technique could apply now, because binding will be a function of the glycan structure."

Similarly, the technique could apply if an antibody targets the glycan moiety of a glycoprotein, he said. But if antibody binding involves the underlying protein structure-as is the case with Arius's AR001 anti$\mathrm{CD} 44 \mathrm{mAb}$ - then the technique would lack the functionality to model that, he said.

Also like Gold, Young thinks the inability to probe mucin signal transduction is a limitation of the mimics' unnatural polymer backbone. It would be important to have mimics that conferred "adhesive capabilities or some other function the cell didn't have before," he said.

"This is a useful research tool that can do certain things, but it is probably an adjunct technique," Young concluded, until the researchers develop mimics that approach the functionality of the native glycoproteins.

\section{Biofriendly is best}

Rabuka said the team is taking the glycopolymer technology one step at a time. "Currently we are only interested in mimicking and studying cell-surface behavior, which is itself a very daunting task," he said.

Nevertheless, Rabuka said, there is no reason to think the technique could not be used to mimic other glycoproteins. "The main hurdles would be in the chemistry" required to synthesize such mimics, he said.

Since submitting the JACS paper, Rabuka said the research team has explored other synthetic backbones and is working to incorporate tri- and tetrasaccharide glycans into the mimics.

"We don't think we'll need to go any larger than that because this is the size of the epitopes of biological interest," he said.

Rabuka added that the team has also extended its studies to other cell types, including cells that do express mucins, and obtained results comparable to those reported in JACS. Results from the experiments with different glycopolymer structures and in different cell types will be published in forthcoming papers, he said.

The team has no immediate plans to patent or out-license its findings, though the researchers might reconsider "after making the backbone more 'biofriendly"' Rabuka told SciBX.

Arius's Young said the research team appeared to be taking their technique down the right path.

"Carbohydrates are complex and there is a lack of tools to study that complexity. Cancer cells put carbohydrates on their surfaces, but we don't know why, what function they have" he said. "To understand why, we need to build up a set of tools" the one Bertozzi's team is developing.

\section{REFERENCES}

1. Singh, P. et al. Trends Cell Biol. 16, 467-476 (2006)

2. Rabuka, D. et al. J. Amer. Chem. Soc.; published online April 11, 2008; 
doi:10.1021/ja710644g

Contact: Carolyn R. Bertozzi, Lawrence Berkeley National Laboratory, Berkeley, Calif.

e-mail: crb@berkeley.edu

3. Rabuka, D. et al. J. Amer. Chem. Soc. 129, 5462-5471 (2007)

4. Paulick, M. et al. Proc. Natl. Acad. Sci. USA 104, 20332-20337 (2007)

COMPANIES AND INSTITUTIONS MENTIONED

Arius Research Inc. (TSX:ARI), Toronto, Ontario, Canada
Eli Lilly and Co. (NYSE:LLY), Indianapolis, Ind.

Fresenius SE (Xetra:FRE), Bad Homburg, Germany Garden State Cancer Center, Belleville, N.J.

Howard Hughes Medical Institute, Chevy Chase, Md. Immunomedics Inc. (NASDAQ:IMMU), Morris Plains, N.J.

Lawrence Berkeley National Laboratory, Berkeley, Calif.

Trion Pharma GmbH, Munich, Germany

University of California, Berkeley, Calif. 